\title{
Notes on survey-based spatial indicators for monitoring fish populations
}

\author{
Mathieu Woillez ${ }^{1, a}$, Jacques Rivoirard ${ }^{2}$ and Pierre Petitgas ${ }^{1}$ \\ IFREMER, Département EMH, BP 21105, 44311 Nantes Cedex 03, France \\ 2 Mines-ParisTech, Centre de Géosciences/Géostatistique, 35 rue St Honoré, 77300 Fontainebleau, France
}

Received 30 June 2008; Accepted 23 January 2009

\begin{abstract}
This paper presents the spatial indicators used in the European project FISBOAT. These are statistics intended to capture spatial patterns of fish populations, using fish density data collected during scientific surveys. To handle diffuse population limits, indicators are designed not to depend on arbitrary delineation of the domain. They characterize the location (centre of gravity and spatial patches), occupation of space (inertia, isotropy, positive area, spreading area and equivalent area) and microstructure. Collocation between different populations is summarized by a global index of collocation. These spatial indicators have the potential to be used in a monitoring system to detect changes in spatial distribution. They could be helpful for relating the spatial distribution properties of fish stocks to their dynamics, their habitats, or to climate change.
\end{abstract}

Key words: Spatial indicators / Monitoring / Spatial patterns / Fish populations

\section{Introduction}

Survey data obtained from monitoring exploited populations provides rare opportunities for ecological investigations of relationships between spatial pattern and population dynamics (MacCall 1990). Spatial indicators are statistics that aim to describe and summarize the spatial distribution of populations (in terms of fish density, location or possibly environmental variables such as depth). They are useful for investigating such relationships and making fishery-independent diagnostics by an indicator-based approach. This paper, designed as a manual, proposes a list of ten spatial indicators (Table 1) to characterise the location (centre of gravity and spatial patches), occupation of space (inertia, isotropy, positive area, spreading area and equivalent area), fine scale structure (microstructure) and overlap between populations (global index of collocation). The list does not, of course, intend to be either fixed or exhaustive. Although some other indicators might also appear to be useful (e.g. the mean depth of the population, other ways to measure patchiness, etc), certain that have been proposed are in fact related. In particular, formal relationships exist between some occupation indicators (Woillez et al. 2007). While such indicators present some redundancy, it may be desirable to maximise the number of indicators in a indicator-based monitoring approach as they increase the opportunities of picking up changes in critical factors over time, compared with the use of prior decisions on these factors (Caddy et al. 2005). In addition, they

\footnotetext{
a Corresponding author: mathieu.woillez@ifremer.fr
}

can be helpful in identifying the spatial dynamic model of a fish population as highlighted by Petitgas (1998).

The selected indicators are documented in a standardised format (Halliday and Mohn 2001) covering several aspects: description, stock attributes, derivation, interpretability, measurability, sensitivity and examples of their use. The measurability section concerns the statistical properties of the estimator of the indicator obtained from the sample values, e.g. variability, bias, skewness, sensitivity to high values, etc. In contrast, the sensitivity section refers specifically to how rapidly the indicator responds to changes in stock status. Remarks that are common to all indicators, for instance on reference points, are made at the end of this document. In addition, R-script functions that have been written to compute spatial indicators, are available in electronic form (see Appendix S1). For a specific application of these indicators to a common stock, readers can refer to the article by Woillez et al. (2007) on hake in the Bay of Biscay.

\section{Theoretical framework for spatial indicators}

When selecting spatial indicators for this manual, care was taken to exclude statistics that would depend on the inclusion or exclusion of zero density values, as such dependence requires the perimeter of the domain to be defined a priori, and perhaps arbitrarily. For instance, the mean of the density values within a given domain is not considered here, nor are the variance or the Gini index (linked to the representation of a 
Table 1. List of the spatial indicators documented and the population characteristics they are thought to be related to.

\begin{tabular}{|c|c|c|c|}
\hline Indicator & Abbrev. & Units or range & Population characteristics \\
\hline Centre of gravity & $C G$ & geographical coordinates & Mean geographic location of the population \\
\hline Inertia & $I$ & square nautical miles & Dispersion of the population around its centre of gravity \\
\hline Anisotropy & $A n$ & $\geqslant 1$ & Elongation of the spatial distribution of the population \\
\hline Isotropy & Is & {$[0,1]$} & Elongation of the spatial distribution of the population \\
\hline Global index of collocation & GIC & {$[0,1]$} & Overlap of two spatial populations \\
\hline Number of spatial patches & $N P$ & $>0$ & Patchiness \\
\hline Positive area & $P A$ & square nautical miles & Area of presence occupied by the stock, even with a low density \\
\hline Spreading area & $S A$ & square nautical miles & $\begin{array}{l}\text { A measure of the area occupied by the stock that takes into } \\
\text { account variations in fish density. }\end{array}$ \\
\hline Equivalent area & $E A$ & square nautical miles & An individual-based measure of the area occupied by the stock \\
\hline Microstructure index & $M$ & {$[0,1]$} & The fine-scale variability of the fish density surface \\
\hline
\end{tabular}

histogram through its concentration Lorenz curve, e.g. Kendall and Stuart 1977, p 49) that measure the statistical dispersion of these values. In contrast, the statistics that were selected here do not depend on whether or not zero density values are included; as such values make a null contribution to the statistics. For instance, the centre of gravity, or mean location of a population, will depend on whether the density value at a sampled location is zero or not, but if it is zero, its numerical contribution to the centre of gravity will be zero.

In particular, the contribution of zero density values is zero in all statistics based on individuals in the population, e.g. the mean location of a population, which is the mean location of the individuals that constitute this population (in such a case the statistics are weighted by the fish density). In contrast, the positive area concerns the area where the fish density values are strictly positive, but does not depend on the level of these density values, that is, on each individual.

Some of the selected statistics (e.g. the centre of gravity) would change if the fish density values were permuted between sampled locations (even assuming a regular sample grid). The other statistics would be unchanged. For instance, the positive area measures the domain covered by the non-zero density values, not its shape, and it would be unchanged by permuting density values. Similarly, the spreading area or the equivalent area will depend on the histogram of density values, not on their location (at least assuming a regular sample grid). As a consequence, these statistics are not dependent on the largescale spatial structure. However they do depend on the fine scale structure through the "support", that is, the surface in terms of size and geometry (e.g. the trawled area) on which each fish density is measured.

\section{Spatial indicators}

\subsection{Centre of gravity}

The centre of gravity $(C G)$ is the mean location of the population, that is, the mean of the location of the individuals that compose it (Bez 1997; Bez and Rivoirard 2001).

\section{Stock attribute}

Mean geographic location of the population.

\section{Derivation}

Let $x$ be a point in two-dimensional space (short for the usual two-dimension notation $(x, y))$, and $z(x)$ be the population density at location $x$. Then, the total abundance of the population is:

$$
Q=\int z(x) \mathrm{d} x,
$$

and the probability density function of the location $\underline{x}$ of a random individual is $z(x) / Q$. The centre of gravity $(C G)$ is:

$$
C G=E(\underline{x})=\int x \frac{z(x)}{Q} \mathrm{~d} x=\frac{\int x z(x) \mathrm{d} x}{\int z(x) \mathrm{d} x} .
$$

In practice, this statistic is estimated from the data through discrete summations over sample locations. In the case of irregular sampling, areas of influence around samples can be used as weighting factors (Fig. 1). Practically, from sample values $z_{i}$ at locations $x_{i}$, with areas of influence $s_{i}$, we have:

$$
C G=\frac{\sum_{i=1}^{N} x_{i} s_{i} z_{i}}{\sum_{i=1}^{N} s_{i} z_{i}} .
$$

\section{Interpretability}

The $C G$ indicates the mean location of the surveyed population. Note that fish may not be present at the $C G$ location (which may be on land, e.g. on an island). Note also that part of the population may be not represented, when not covered geographically by the sampling. To ensure that displacements of $C G$ over time are not due to changes in the sampling design (e.g. due to bad weather), the $C G$ of the sample locations (unweighted by fish density) can also be produced.

\section{Measurability}

The estimated $C G$ is sensitive to high fish density values. It may differ from the true unknown $C G$, particularly when high density values exist (whether sampled or not). Resampling can be considered to evaluate the sensitivity of the estimated $C G$ to sample values (Woillez 2007). 


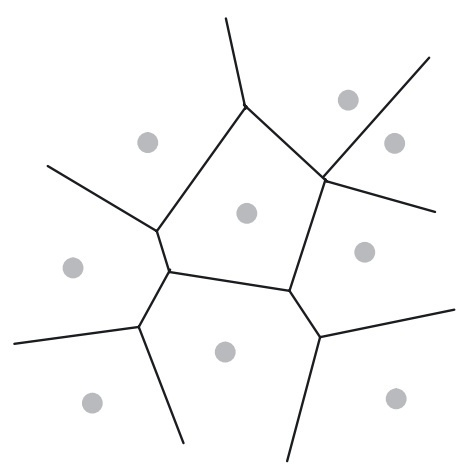

Fig. 1. Area of influence (delimited by the black lines) of sample location (in grey) is defined as the area made up of the points in space that are closer to this sample than to others. It can be evaluated by overlaying a very fine regular grid and counting the grid points closer to the sample. Known or supposed boundaries (e.g. land, a limit distance of influence from a sample location) of the sampled population may be used.

\section{Sensitivity}

Despite the possible difference between true and estimated $C G$, a shift during a series, when it is gradual, is likely to represent an actual shift of the population. On the other hand, an eccentric estimated $C G$ requires a visual inspection of the fish density to detect the causes (e.g. unusual presence of high density values in some remote area, or disappearance of usually high values in a particular region).

\section{Examples}

Previous authors have used the centre of gravity, also referred to as the distributional centroï, to describe the distribution of a population in cases including walleye pollock eggs and larvae (Kendall and Picquelle 1990), Pacific hake larvae (Hollowed 1992), cod off Newfoundland (Atkinson et al. 1997), yellowtail flounder off the Grand Bank (Brodie et al. 1998), European hake eggs and larvae (Alvarez et al. 2001), and fish-at-age (Woillez et al. 2007) in the Bay of Biscay. For example, the $C G$ s have been used to describe the distribution of strong year classes of Pacific hake late stage larvae, and also the systematic shift towards the south east in cod off Newfoundland from 1987 to 1993. Seasonal changes in distributions have also been described, e.g. for eggs and larvae of European hake in the Bay of Biscay and of walleye pollock.

\subsection{Inertia}

The inertia is the variance of the location of individuals in the population, that is, the mean square distance between an individual fish and the centre of gravity of the population (Bez 1997).

\section{Stock attribute}

Inertia describes the dispersion of the population around its centre of gravity.

\section{Derivation}

With the notations used for $C G$, the inertia $(I)$ is

$$
I=\operatorname{Var}(\underline{x})=\frac{\int(x-C G)^{2} z(x) \mathrm{d} x}{\int z(x) \mathrm{d} x},
$$

and is estimated as:

$$
I=\frac{\sum_{i=1}^{N}\left(x_{i}-C G\right)^{2} s_{i} z_{i}}{\sum_{i=1}^{N} s_{i} z_{i}}
$$

\section{Interpretability}

The inertia $I$ indicates how dispersed the population is around its centre of gravity.

\section{Measurability}

Inertia is sensitive to high density values. $I$ is measured in square nautical miles (in 2D). The square root of $I$, which is the standard deviation of locations of individuals (root mean square distance between individuals and their $C G$ ), may be preferred, as this is measured in nautical miles. The sensitivity of inertia to sample values can be explored by resampling (Woillez 2007).

\section{Sensitivity}

An increase in $I$ indicate that a population is more dispersed around its $C G$, which is to say high density values are more scattered. Although the population is then scattered over a larger region, the actual area covered by the population may be smaller (see the different area indicators).

\section{Examples}

Most of the references cited on CGs have also described the studied population in terms of inertia, or they represent it graphically as an ellipse (see next section on isotropy). Brodie et al. (1998) showed a decrease in the area of the ellipse for the yellowtail flounder of the Grand bank in late 1980s after a period of stability. Atkinson et al. (1997) showed a shift of the $C G$, on the northern cod population, accompanied by a decrease in the size of the ellipse, which is the inertia. For hake eggs in the Bay of Biscay, the size of the ellipses increased somewhat from February to May in both directions, N-S and W-E (Alvarez et al. 2001), while the inertia of fish increased with age (Woillez et al. 2007). 

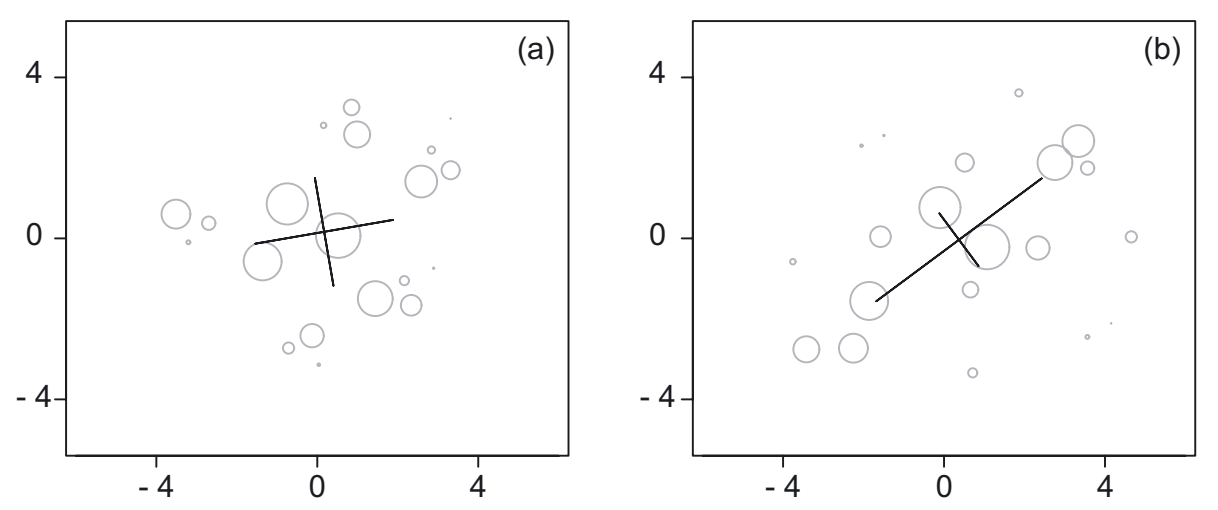

Fig. 2. Two examples of spatially distributed data sets, with the second case (b) showing more marked anisotropy than the first one (a). The black cross is positioned on the centre of gravity, from which it represents the square root of inertia along each of the two principal directions.

\subsection{Isotropy and anisotropy}

When the dispersion of the population around its centre of gravity is the same along every direction, the spatial distribution is said to be isotropic. In general, the dispersion of a population around its centre of gravity is not identical in every spatial direction: there is an anisotropy. The root mean square distance to the centre of gravity is maximal along the first principal axis, and minimal along the second principal axis, orthogonal to the first one (in 2D). The anisotropy index is taken as the ratio between these distances, and the isotropy index as the inverse ratio.

\section{Stock attribute}

Anisotropy measures the elongation of the spatial distribution of the population.

\section{Derivation}

In two dimensions, the total inertia of a population can be decomposed on its two principal axes, orthogonal to each other, explaining respectively the maximum and the minimum of the inertia. These two principal axes and their inertia can be obtained as the eigen vectors and values of a principal component analysis of the coordinates of individuals in the population (i.e. the coordinates of the samples weighted by fish densities) (Bez 1997). The square root of the inertia along a given axis (or root mean square distance to $C G$ ) gives the standard deviation of the projection of the location of the population along that axis. These can be represented conveniently on a map with a cross depicting the two principal directions (Fig. 2), or with an ellipse (with area proportional to the total inertia). The anisotropy index $(\geqslant 1)$ is the square root ratio between the maximum and the minimum of the inertia. Similarly, an index of isotropy can be defined as the inverse of anisotropy, ranging more conveniently from 0 to 1 :

$$
\text { Isotropy }=\sqrt{\frac{I \min }{I \max }} \text { and Anisotropy }=\sqrt{\frac{I \max }{I \min }} .
$$

\section{Interpretability}

The anisotropy index roughly gives the elongation of the population in space. It does not take into account the actual shape of the distribution, which may differ from elliptical or may be made up by a number of different patches.

\section{Measurability}

The anisotropy and isotropy indicators are equivalent, being the inverse of each other. However, since the anisotropy is unbounded above 1, the isotropy is more robust and may be more conveniently used, e.g. in correlation or regression analyses. In case of isotropy, that is, when the anisotropy and isotropy approach 1 , the directions of the principal axes, orthogonal to each other, become arbitrary. In addition, sensitivity to sample values can be examined by resampling as illustrated in Woillez (2007).

\section{Sensitivity}

Sudden changes in anisotropy index may be due to the disappearance or, inversely, the appearance of patches of fish in some areas.

\section{Examples}

Few authors have discussed anisotropy even when they showed it in their figures. In Alvarez et al. (2001), the direction of the principal axis of hake egg distribution in the Bay of Biscay corresponds to that of the shelf break, i.e. NW-SE, throughout the whole sampling period. Woillez et al. (2007) completed the description for the later fish stages, showing that a preferential direction was more marked for age 0 and age $5+$. The direction for age group 0 corresponds roughly to muddy sediment off Brittany. For ages 4 and 5+, the direction corresponds to the shelf edge, where most older hake are concentrated. For the intermediate ages, the population is still anisotropic, probably because of the general shape of the continental shelf, but the anisotropy is less marked. 


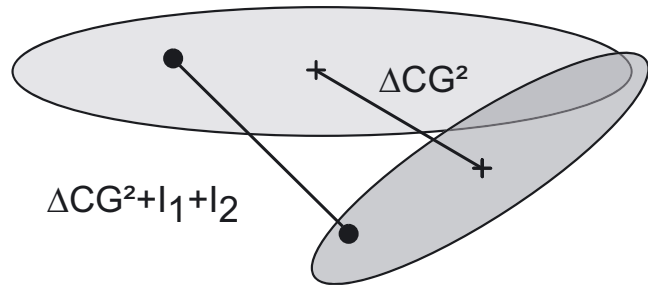

Fig. 3. Collocation of two spatial populations, represented here by two ellipses, with their centre of gravity, is measured with the global index of collocation through specific distances.

\subsection{Global index of collocation}

The global index of collocation looks at the extent to which two populations are geographically distinct, by comparing the distance between their $C G$ s and the mean distance between individual fish taken at random and independently from each population (Bez and Rivoirard 2000).

\section{Stock attribute}

Spatial overlap of two populations.

\section{Derivation}

Let us consider two populations with densities $z_{1}(x)$ and $z_{2}(x)$ at point $x$, with $\Delta C G$ being the distance between their centres of gravity, and $I_{1}$ and $I_{2}$ their respective inertias (Fig. 3). The mean square distance between individuals taken at random and independently from each population is $\Delta C G^{2}+I_{1}+I_{2}$ and the global index of collocation $(G I C)$ is:

$$
G I C=1-\frac{\Delta C G^{2}}{\Delta C G^{2}+I_{1}+I_{2}},
$$

or 1 if $\Delta C G^{2}=I_{1}=I_{2}=0$. The $G I C$ indicator ranges between 0 , in the extreme case where each population is concentrated on a single but different location (inertia $=0, \Delta C G^{2}>0$ ), and 1, where the two $C G$ s coincide.

\section{Interpretability}

Collocation is considered here on a population scale, that is to say that the populations are grossly in the same geographic place but not necessarily present at the same sample locations. A spatial distribution of a population that were distributed all around another one, with the same $C G$, would give a GIC equal to 1 , even if the second population was not overlapping the first population locally. Local overlapping between two populations should rather be addressed using the local index of collocation, that is, the non-centred correlation between their fish densities.

\section{Measurability}

Alternative indicators, also between 0 and 1 , are given by $\sqrt{G I C}$ for collocation, or $\sqrt{1-G I C}$ for separation (ratio of distance between the $C G$ s to distance between individuals from the two populations).

\section{Sensitivity}

Unusually high GIC requires inspection of the fish density data.

\section{Examples}

In Bez and Rivoirard (2000), global and local collocation indices were measured on egg densities of pelagic species in the Bay of Biscay in 1998. Large global collocation exists between anchovy and horse mackerel, while sardine and mackerel are geographically distinct. Local collocation appears very small between mackerel and the other species (anchovy, sardine and horse mackerel). In Woillez et al. (2007), GIC was used to detect outliers in the age time series of hake in the Bay of Biscay. The year 2000 is notable different for age 0 .

\subsection{Number of spatial patches}

A population of fish may be distributed into several spatial patches, much larger in size than a fish school. An algorithm has been written to identify patches (Woillez et al. 2007) by attributing each sample to the nearest patch, with respect to a maximal threshold distance to its $C G$.

\section{Stock attribute}

Patchiness.

\section{Derivation}

The algorithm starts from the sample value displaying the maximum density $z(x)$, and considers every other sample in decreasing order of density. The maximum value initiates the first patch (Fig. 4a). Then, the current sample value is attributed to the nearest patch, if the distance to its $C G$ is smaller than the threshold distance dlim (Fig. 4b). Otherwise, the current sample value defines a new patch (Fig. 4c). Spatial patches whose abundance is $>10 \%$ of overall abundance are retained. The summary index is then the number of spatial patches (Fig. 4d).

\section{Interpretability}

The number of spatial patches indicates the level of patchiness of the distribution of a fish population at a large scale. 


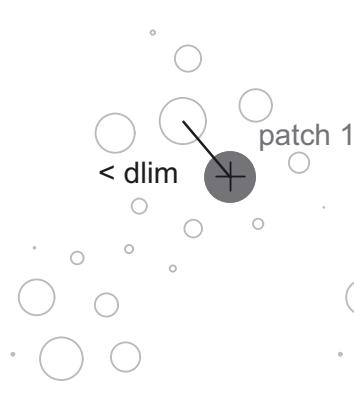

(a)

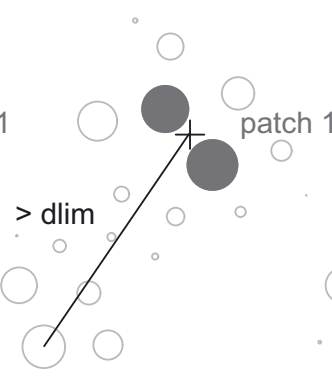

(b)

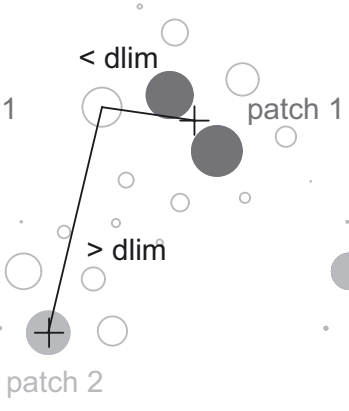

(c)

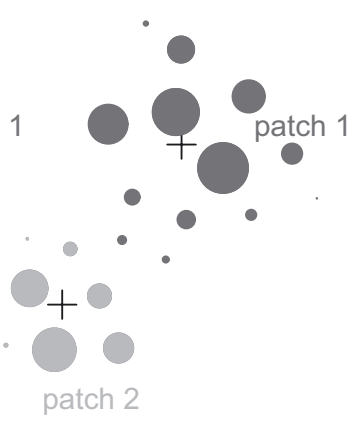

(d)

Fig. 4. Main steps of the algorithm used to determine the number of spatial patches of a population, where the current sample value is attributed to the nearest patch, if the distance to its $C G$ is smaller than the threshold distance dlim.
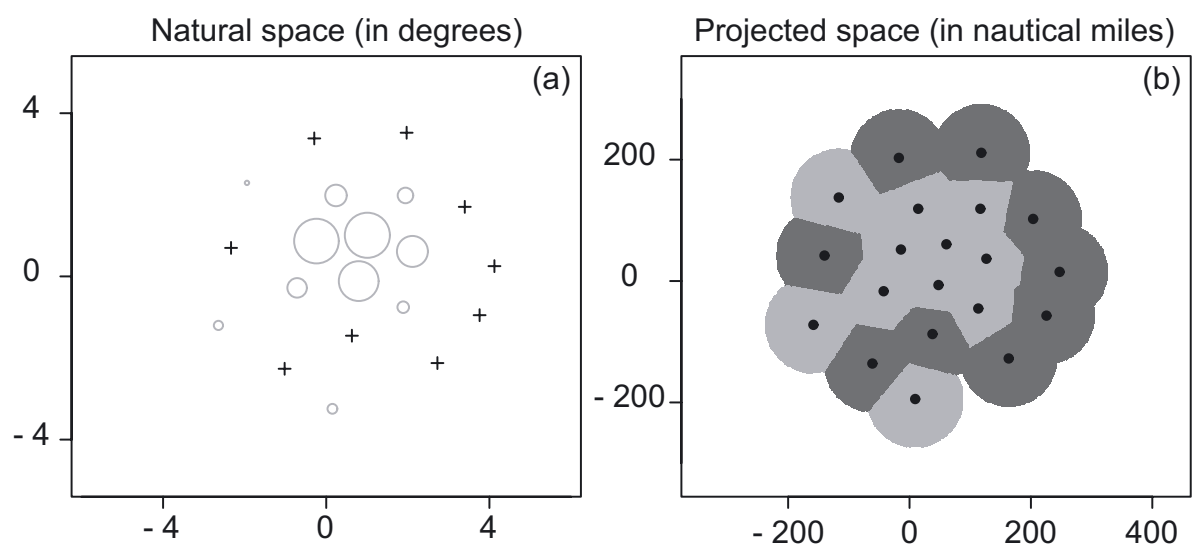

Fig. 5. Bubble plot of the sample values (a) and corresponding positive area (b) shaded in light grey (with a limit to the area of influence of each sample). The projection used multiplies longitudes by $60 \times$ cosine of the mean latitude (here 0 degree), and latitudes by 60 .

\section{Measurability}

The identification of spatial patches is dependent on the threshold distance dlim, typically some fraction of the diameter of the sampled domain, chosen by the user. The number of spatial patches is very sensitive to the location of the highest fish density values, as one would expect.

\section{Sensitivity}

The location of spatial patches is likely to present some stability in time. Hence a change in the number of spatial patches is likely to reveal the disappearance, or the appearance of fish in some areas.

\section{Examples}

In Woillez et al. (2007), the number of spatial patches has been illustrated on hake in the Bay of Biscay. It increases slightly up to age 3 then decreases for older ages. Disappearance of patches has been observed and localised for age 0 hake, in particular for the year 2000.

\subsection{Positive area}

The positive area is the measure, in square nautical miles, of the space occupied by fish densities strictly above zero (Woillez et al. 2007).

\section{Stock attribute}

Area of presence occupied by the stock, even with a low density.

\section{Derivation}

The positive area $(P A)$ is estimated from data as the sum of the areas of influence around samples where there are fish densities $>0$ (Fig. 5):

$$
\mathrm{PA}=\sum_{i} s_{i} 1_{z_{i}>0} .
$$

\section{Interpretability}

The positive area measures the area of effective presence, in square nautical miles. It does not include zero density areas 
possibly existing between positive density areas, and it may correspond to a small fraction of the geographical envelope of fish presence, in particular when the inertia is high.

\section{Measurability}

Zero values of density make no contribution to the positive area. However, the positive area is sensitive as much to the low values of density as to the high values of density.

\section{Sensitivity}

Changes in the positive area may reveal changes in the way the population occupies space. Numerous low fish density values will impact the positive area, even though they contribute little to the global abundance.

\section{Examples}

In Woillez et al. (2007), positive area of hake in the Bay of Biscay was relatively stable until age 3 and then dropped. It was also shown that, whereas positive area decreased with age, inertia increased with age: the older hake occupying a smaller but more dispersed area.

\subsection{Spreading area}

The spreading area $(S A)$ is a measure, in square nautical miles, of how the population is distributed in space, taking into account the variations in fish density (Woillez et al. 2007).

\section{Stock attribute}

A measure of the area occupied by the stock that considers variations in fish density.

\section{Derivation}

Let $T$ be the cumulative area occupied by the density values, ranked in decreasing order; $Q(T)$ be the corresponding cumulative abundance; and $Q$ be the overall abundance. The $S A$ (expressed in square nautical miles) is then simply defined as twice the area below the curve expressing $(Q-Q(T)) / Q$ as a function of $T$ (Fig. 6):

$$
S A=2 \int \frac{Q-Q(T)}{Q} \mathrm{~d} T .
$$

As $(Q-Q(T)) / Q$ decreases from 1 to 0 and is convex, the $S A$ is less than the positive area. It equals the positive area when the population is evenly spread with a constant density. The curve in Figure 6, is a derivation of the Lorenz curve representing the histogram of fish density values, but it has the advantage of receiving no contribution from zero density values. The spreading area can be related to the area occupied by the positive fish density values $P A$ and their Gini index of dispersion $G_{0}$ through $\frac{S A}{P A}+G_{0}=1$ (Woillez et al. 2007).

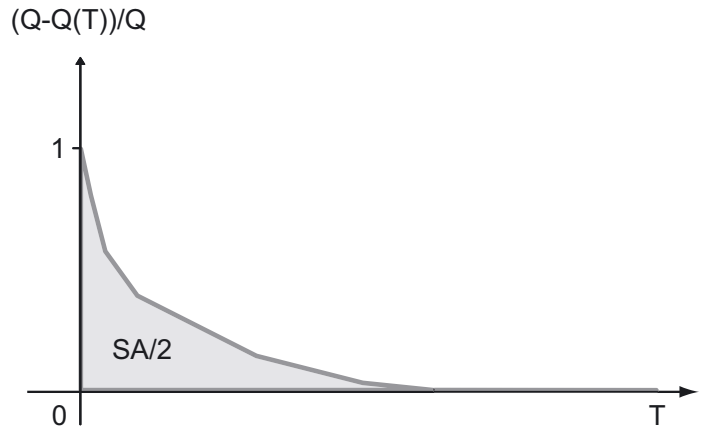

Fig. 6. The spreading area $(S A)$ is defined as twice the area below the curve expressing $(Q-Q(T)) / Q$ as a function of $T$.

\section{Interpretability}

Fish abundance is generally distributed in space with highly varying fish density values, spreading over its positive area. The spreading area index was designed to provide a measure of the area occupied by the population, taking into account such variations in fish density while satisfying the condition of having no contribution from zero density values. The spreading area depends exclusively on the amount and histogram of positive fish density values.

\section{Measurability}

Zero values of density make no contribution to the spreading area, in contrast to other indicators that characterise aggregation (area coverage: Swain and Sinclair 1994; Gini index: Myers and Cadigan 1995; spatial selectivity index: Petitgas 1998). Therefore, in the calculation of the spreading index, the delineation of the data positive domain is not necessary. The spreading area depends on the variation in density values (and not on the absolute abundance) and is much less sensitive to low values of density than is the positive area.

\section{Sensitivity}

Changes in $S A$ are likely to reveal changes in the way the abundance is split into low and high density values.

\section{Examples}

In Woillez et al. (2007), the hake population in the Bay of Biscay was described using $S A$. This showed a higher $S A$ of 3 year-old hake. In addition, a decrease of $S A$ through the time series was detected for hake of ages 4 and 5+.

\subsection{Equivalent area}

The equivalent area represents the area, in square nautical miles, that would be covered by the population if all individuals had the same density, equal to the mean density per individual (Bez and Rivoirard 2001). 


\section{Stock attribute}

An individual-based measure of the area occupied by the stock.

\section{Derivation}

The transitive geostatistical approach (Matheron 1971) can be used to describe the spatial distribution of a fish population when it includes a few large values of density, and when it is difficult to delimit a domain with homogeneous variations. The spatial structure is then represented by a (transitive) covariogram, a function of the distance between two locations:

$$
g(h)=\int z(x) z(x+h) \mathrm{d} x .
$$

Here, the equivalent area $(E A)$ is defined as the integral range of the covariogram:

$$
E A=\frac{\int g(h) d h}{g(0)}=\frac{Q^{2}}{g(0)}=\frac{Q^{2}}{\int z(x)^{2} \mathrm{~d} x}=\frac{\left(\int z(x) \mathrm{d} x\right)^{2}}{\int z(x)^{2} \mathrm{~d} x} .
$$

It can also be written:

$$
E A=\frac{Q}{\int z(x) \frac{z(x)}{Q} \mathrm{~d} x} .
$$

The $E A$ is a ratio of the total abundance to the mean density per individual (the denominator in the previous equation (Fig. 7)). Practically, in the discrete case with sample values $z_{i}$ and areas of influences $s_{i}$, this can be written:

$$
E A=\frac{\left(\sum_{i=1}^{N} s_{i} z_{i}\right)^{2}}{\sum_{i=1}^{N} s_{i} z_{i}^{2}} .
$$

The $E A$ ranges from 0 to the positive area. It would be equal to the positive area if all strictly positive values of density were the same. The equivalent area can be related to the area occupied by the positive fish density values $P A$ and their coefficient of variation $C V_{0}$ through $\frac{P A}{E A}=1+C V_{0}^{2}$. The equivalent area $E A$ and spreading area $S A$ are related through inequalities, in particular $E A \leqslant \frac{9}{8} S A$ (Woillez et al. 2007).

\section{Interpretability}

The positive area describes the area of presence of fish, with a low density value being equivalent to a high one. The spreading area describes the area occupied by the stock, taking into account the variations in fish density. Now, the equivalent area is yet another way to do this, but is individual-based and therefore gives the same weight to each individual: the weight of a sample is proportional to its fish density. Like the spreading area, the equivalent area depends exclusively on the amount and histogram of positive fish density values.

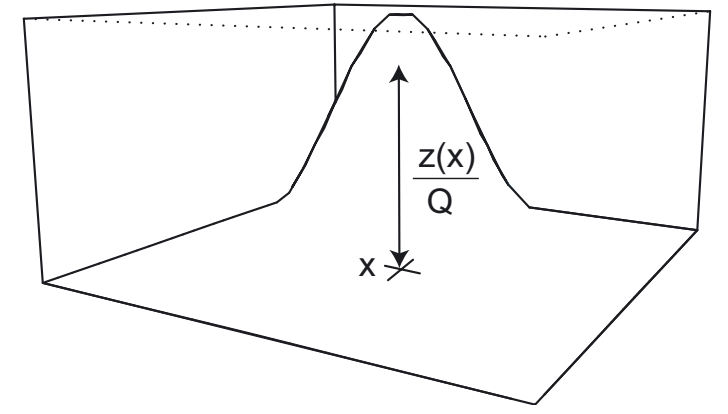

Fig. 7. The probability density function for a random individual to be at $x$ is given by $z(x) / Q$.

\section{Measurability}

The equivalent area is independent of the absolute abundance. Being individual-based, it is very sensitive to the highest density values. The inverse of the equivalent area can be considered as an index of aggregation (Bez and Rivoirard 2001).

\section{Sensitivity}

Changes in $E A$ are likely to reveal changes in the contribution of high density values to the total abundance.

\section{Examples}

In Woillez et al. (2007), the equivalent area for hake in the Bay of Biscay was shown to be larger in average for 3-year-old hake than for other ages in the range $0-5+$.

\subsection{Microstructure index}

The microstructure index (Woillez et al. 2007) measures the relative importance of structural components that have a smaller scale than the sample lag (including random noise).

\section{Stock attribute}

The fine-scale variability of the fish density surface.

\section{Derivation}

The microstructure index $(M I)$ is taken as the relative decrease of the transitive covariogram (Matheron 1971; Bez et al. 1997) between distance zero and a distance $h_{0}$ chosen to represent the mean lag between samples (Fig. 8):

$$
M I=\frac{\left(g(0)-g\left(h_{0}\right)\right)}{g(0)} .
$$

It lies between 0 and 1 . Values close to 0 correspond to a very regular, well-structured density surface, and values close to 1 correspond to a highly irregular, poorly structured density surface. 


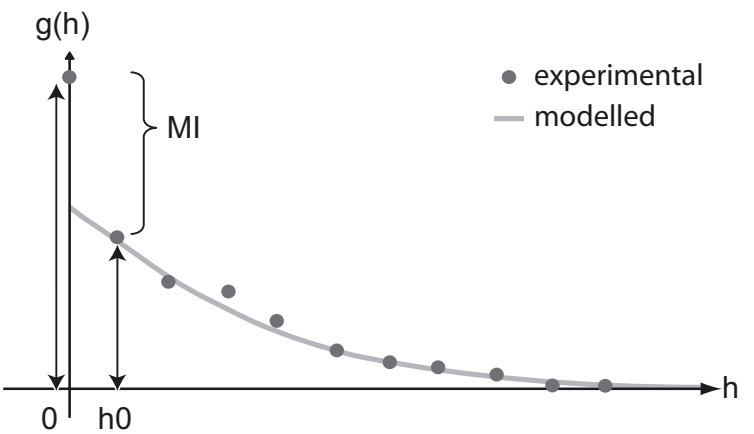

Fig. 8. Experimental and modelled covariogram with the representation of the microstructure index.

\section{Interpretability}

The microstructure index measures how irregular the fish density surface is on the fine scale. However, it does not make a distinction between spatial variability with a lesser range than the chosen lag, but positive, and purely random variability (e.g. due to noise or sampling error).

\section{Measurability}

The microstructure index is very sensitive to high fish density values. However, as it is derived from the transitive covariogram, it is more robust than would be a similar index obtained from the more traditional variogram or covariance.

\section{Sensitivity}

A high microstructure index is likely to correspond to predominant fine-scale aggregations of fish in the population.

\section{Examples}

In Woillez et al. (2007), microstructure index was followed through age and time on the hake population in the Bay of Biscay. It showed a relative stability for the younger ages, then it rose markedly from age 4 to age $5+$.

\section{Reference points}

As the spatial distribution of a population in a pristine state is generally not known and does not correspond to the one of the early years of the monitoring time series, the reference points of the spatial indicators can only be determined by examining their evolution in time. A period of acceptable state of the spatial distribution of the population has to be determined according to the indicator values (some stability of the indicator is expected along the series) and broader knowledge, such as the level of fish abundance.

\section{Conclusion}

This paper presents a non exhaustive list of spatial indicators that are useful to capture spatial patterns of a fish population from research survey data. Such indicators, as well as more traditional biological indicators, can form an important part of a fishery monitoring system, provided that appropriate statistical methods for assessing and interpreting trends are used in order to assess the state of a stock. Multivariate methods, as well as quality process control, might be needed, either to combine information or to monitor the indicator time-series by triggering alarms on stock status with assigned risks of false alarm. Spatial indicators allow changes to be detected in the spatial distribution of a fish population. They could be helpful in relating the spatial distribution properties of fish stocks to their dynamics. Their use might contribute to our understanding of the impacts of climate change and fishing on fish stocks, and to making fishery-independent diagnostics based on an indicator-based approach.

Acknowledgements. The study was carried out with the financial support of the European Union (project FISBOAT - Fisheries Independent Survey Based Operational Assessment Tools, DG-Fish, $6^{\text {th }}$ Framework STREP, Contract 502572).

\section{Supporting information}

\section{Appendix S1}

R-script functions, named Spatial_indicators_functions.r, for computing spatial indicators. These functions compute the ten selected indicators with standard R statements (no additional package is needed); the basic data requirement is a set of vectors with longitude, latitude and fish density. Supporting Information is only available in electronic form at www.alr-journal.org.

\section{References}

Alvarez P., Motos L., Uriarte A., Egana J., 2001, Spatial and temporal distribution of European hake, Merluccius merluccius (L.), eggs and larvae in relation to hydrographical conditions in the Bay of Biscay. Fish. Res. 50, 111-128.

Atkinson D.B., Rose G.A., Murphy E. F., Bishop C.A., 1997, Distribution changes and abundance of northern cod (Gadus morhua), 1981-1993. Can. J. Fish. Aquat. Sci. 54 (Suppl. 1), 132138.

Bez N., 1997, Statistiques individuelles et géostatistique transitive en écologie halieutique. Thèse de dr Géostatistique, Ecole Nationale Supérieure des Mines de Paris.

Bez N., Rivoirard J., 2000, Indices of collocation between populations. In: Checkley D.M., Hunter J.R., Motos L., von der Lingen C.D. (Eds.). Workshop on the Use of Continuous Underway Fish Egg Sampler (CUFES) for mapping spawning habitat of pelagic fish. GLOBEC Rep., pp. 48-52.

Bez N., Rivoirard J., 2001, Transitive geostatistics to characterize spatial aggregation with diffuse limits: an application on mackerel ichthyoplankton. Fish. Res. 50, 41-58. 
Bez N., Rivoirard J., Guiblin P., Walsh M., 1997, Covariogram and related tools for structural analysis of fish survey data. In: Baafi E.Y., Schofield N.A. (Eds.). Geostatistics Wollongong'96 2, pp. 1316-1327.

Brodie W.B., Walsh S.J., Atkinson D.B., 1998, The effect of stock abundance on range contraction of yellowtail flounder (Pleuronectes ferruginea) on the Grand Bank of Newfoundland in the Northwest Atlantic from 1975 to 1995. J. Sea Res. 39, 139152.

Caddy J.F., Wade E., Surette T., Hebert M., Moriyasu M., 2005, Using an empirical traffic light procedure for monitoring and forecasting in the Gulf of St. Lawrence fishery for the snow crab, Chionoecetes opilio. Fish. Res. 76, 123-145.

Halliday R.G., Mohn R., 2001, Proceedings of the Fisheries Management Studies working group, 8-11 January 2001. Canadian Science Advisory Secretariat, Proc. Ser. 2001/08.

Hollowed A.B., 1992, Spatial and temporal distribution of Pacific hake, Merluccius productus, larvae and estimates of survival during early life stages. CalCOFI Rep. 33, pp. 100-123.

Kendall A.W., Picquelle S.J., 1990, Egg and larva distributions of walleye pollock Theragra chalcogramma in Shelikof Strait, Gulf of Alaska. Fish. Bull. 88, 133-154.

Kendall M., Stuart A., 1977, The advanced theory of statistics, Vol. 1, 4th edn. Charles Griffin, London.
MacCall A.D., 1990, Dynamic Geography of Marine Fish Populations. University of Washington Press, Seattle.

Matheron G., 1971, The theory of regionalized variables and its applications. Les cahiers du Centre de Morphologie Mathématique, Fasc. 5. Ecole Nationale Supérieure des Mines de Paris.

Myers R., Cadigan N., 1995, Was an increase in natural mortality responsible for the collapse of northern cod? Can. J. Fish. Aquat. Sci. 52, 1274-1285.

Petitgas P., 1998, Biomass dependent dynamics of fish spatial distributions characterized by geostatistical aggregation curves. ICES J. Mar. Sci. 55, 443-453.

Swain D., Sinclair A., 1994, Fish distribution and catchability: what is the appropriate measure of distribution? Can. J. Fish. Aquat. Sci. 51, 1046-1054.

Woillez M., 2007, Contributions géostatistiques à la biologie halieutique. Thèse de dr Géostatistique, École Nationale Supérieure des Mines de Paris.

Woillez M., Poulard J.C., Rivoirard J., Petitgas P., Bez N., 2007, Indices for capturing spatial patterns and their evolution in time, with application to European hake (Merluccius merluccius) in the Bay of Biscay. ICES J. Mar. Sci. 64, 537-550. 